\title{
Pediatric Radiology Review
}

\author{
M.D. Pappas, L.G. Yamamoto, and O. Anene, eds.
}

Totowa, NJ: Humana Press, 2007, 174 pages, $\$ 90$

Pediatric Radiology Review, written for an audience of nonradiologists (medical students, residents, and clinicians) involved in the medical care of children, consists of 6 sections (chest, lateral neck, abdominal, skull, and long-bone extremity radiographs and head CT). The stated purpose of the book is to help the reader recognize the anatomic details of radiographic images with regard to common clinical problems and to correlate radiographic findings with the principles of embryology, anatomy, and surgery. Ultrasound, MRI, and body CT are not covered.

Overall, the variety and content of the cases chosen are appropriate for the pediatric clinician. The bone radiography section is especially good, stressing the importance of clinical suspicion and of dedicated views. The illustrated dedicated views were also the clearest and sharpest images. The immediate feedback one receives in answering each question on the CD-ROM is effective, as is the more detailed explanation that can be called up for most answers.

The book is limited in that it assumes some prior training in radiographic interpretation. Nonradiologists would be well served by a review of basic radiographic technique and anatomy for each section, as well as an algorithmic approach to the interpretation of each type of radiograph. An understanding of normal findings, and a few common normal variants, would serve as a valuable starting point in each section before pathology is presented. The generous use of arrows would add much to these well-chosen examples (put here to best use in the long-bone section).

More examples of issues in the neonatal and pediatric intensive care unit settings would enrich the chest and abdomen sections of this book. Consideration could be given to limiting the number of skull radiographs and including these in another section. The thoracic and lumbar spine is briefly discussed in the bone section. The head CT section is limited to trauma examples. Only occasional discussion is offered as to when to (and even more important, when not to) order more advanced imaging studies, such as ultrasound, CT, or MRI.

Regarding the ease of use of the text and CD-ROM, some findings are far easier to see on the CD-ROM images (particularly in the chest section). A single zoom view is offered, although no method is provided to window or level the computer images. There are presumably typographic mistakes in the labeling of correct answers on the CD-ROM, especially in the section on CT scan review. There are no images on the CD-ROM for question 42 of the chest section, and the images for questions 62-66 are not of the elbows of a 20-year-old, as stated.

In summary, the title is misleading, as only a small fraction of pediatric imaging is addressed. A title of Pediatric Radiography Case Review would be more accurate. Despite the limitations and problems on the CD-ROM, the book would be useful for medical students, residents, pediatricians, and general practitioners who care for children because it provides a focused examination of radiologic diagnoses and treatments of common pediatric diseases.

\author{
Nancy E. Fitzgerald \\ M.D. Anderson Cancer Center \\ Houston, Texas
}

